\title{
Spatiotemporal Profile of Voltage-Sensitive Dye Responses in the Visual Cortex of Tree Shrews Evoked by Electric Microstimulation of the Dorsal Lateral Geniculate and Pulvinar Nuclei
}

\author{
Matthieu P. Vanni, ${ }^{1}$ Sébastien Thomas, ${ }^{1}$-Heywood M. Petry, ${ }^{2}$ Martha E. Bickford, ${ }^{3}$ and Christian Casanova ${ }^{1}$ \\ ${ }^{1}$ Laboratoire des Neurosciences de la Vision, École d'optométrie, Université de Montréal, Montreal, Quebec H3C 3JC, Canada, and Departments of \\ ${ }^{2}$ Psychological and Brain Sciences and ${ }^{3}$ Anatomical Sciences and Neurobiology, University of Louisville, Louisville, Kentucky 40292
}

The primary visual cortex (V1) receives its main thalamic drive from the dorsal lateral geniculate nucleus (dLGN) through synaptic contacts terminating primarily in cortical layer IV. In contrast, the projections from the pulvinar nucleus to the cortex are less clearly defined. The pulvinar projects predominantly to layer I in V1, and layer IV in extrastriate areas. These projection patterns suggest that the pulvinar nucleus most strongly influences (drives) activity in cortical areas beyond V1. Should this hypothesis be true, one would expect the spatiotemporal responses evoked by pulvinar activation to be different in $\mathrm{V} 1$ and extrastriate areas, reflecting the different connectivity patterns. Weinvestigated this issue by analyzing the spatiotemporal dynamics of cortical visual areas' activity following thalamic electrical microstimulation in tree shrews, using optical imaging and voltage-sensitive dyes. As expected, electrical stimulation of the dLGN induced fast and local responses in V1, as well as in extrastriate and contralateral cortical areas. In contrast, electrical stimulation of the pulvinar induced fast and local responses in extrastriate areas, followed by weak and diffuse activation in $\mathrm{V} 1$ and contralateral cortical areas. This study highlights spatiotemporal cortical activation characteristics induced by stimulation of first (dLGN) and high-order (pulvinar) thalamic nuclei.

Key words: driver; geniculate nucleus; modulator; pulvinar; thalamocortical connections

\section{Significance Statement}

The pulvinar nucleus represents the main extrageniculate thalamic visual structure in higher-order mammals, but its exact role remains enigmatic. The pulvinar receive prominent inputs from virtually all visual cortical areas. Cortico-thalamo-cortical pathways through the pulvinar nuclei may then provide a complementary route for corticocortical information flow. One step toward the understanding of the role of transthalamic corticocortical pathways is to determine the nature of the signals transmitted between the cortex and the thalamus. By performing, for the first time, high spatiotemporal mesoscopic imaging on tree shrews (the primate's closest relative) through the combination of voltage-sensitive dye recordings and brain stimulation, we revealed clear evidence of distinct thalamocortical functional connectivity pattern originating from the geniculate nucleus and the pulvinar nuclei.

\section{Introduction}

The visual nuclei of the dorsal thalamus include the well studied dorsal lateral geniculate nucleus (dLGN), and the more enig-

\footnotetext{
Received Feb. 17, 2015; revised July 4, 2015; accepted July 20, 2015.

Author contributions: M.P.V. and C.C. designed research; M.P.V. performed research; M.P.V., S.T., and C.C. analyzed data; M.P.V., S.T., H.M.P., M.E.B., and C.C. wrote the paper.

This work was supported by NIH Grant EY016155 to M.E.B., H.M.P., and C.C.; FRSQ provided part of C.C.'s salary (National Researchers Program). We thank, Geneviève Cyr, Karine Minville, Martin Villeneuve, Frédéric Chavane, and David Fitzpatrick for their technical assistance, and David Fitzpatrick for providing some tree shrews.

The authors declare no competing financial interests.

Correspondence should be addressed to Dr Matthieu Vanni, Kinsmen Laboratory of Neurological Research, University of British Columbia, Detwiller Pavilion, 2255 Wesbrook Mall, Vancouver, BC V6T 1Z3, Canada. E-mail: mvanni76@gmail.com.
}

matic pulvinar nucleus. In mammals, the dLGN is considered a first-order relay nucleus because it receives direct input from the retina and projects densely to the primary visual cortex (V1). In contrast, the pulvinar nucleus receives little direct input from the retina (Warner et al., 2010), projects sparsely to V1, and densely to multiple extrastriate visual cortical areas (Ogren and Hendrickson, 1977; Abramson and Chalupa, 1985; Rockland et al., 1999; Chomsung et al., 2010). Geniculocortical projections are deemed to relay visual information from the retina and "drive" cortical activity because lesions of the dLGN severely compro- 
mise visually evoked activity in V1 (Malpeli et al., 1981). In contrast, the function of pulvinocortical projections is less clearly defined. Recent studies unambiguously demonstrated that silencing the pulvinar nucleus significantly affects V1 activity patterns in primates and cats (Purushothaman et al., 2012). However, it is unknown whether these effects were chiefly due to silencing of direct pulvinar-V1 projections, or predominately due to more indirect effects of silencing pulvinocortical projections to extrastriate cortical areas which subsequently alter corticocortical connections.

Thus, the fundamental properties of inputs from the pulvinar nucleus to the cortex are still largely unknown. To begin to address this problem, we compared the impact of electrically stimulating either the dLGN or the pulvinar nucleus on V1 activity patterns. To accomplish this, we used voltage-sensitive dye (VSD) imaging (Shoham et al., 1999) and wide-field imaging of $\mathrm{V} 1$ and surrounding extrastriate cortical areas in tree shrews. This allowed us to monitor the intensity of cortical activation, as well as the spatial and temporal patterns of this activation.

\section{Materials and Methods}

Animal preparation. All procedures were made in accordance with the guidelines of the Canadian Council for the Protection of Animals, and the experimental protocol was accepted by the Ethics Committee of the Université de Montréal. Tree shrews of either sex were initially anesthetized with a mixture of ketamine hydrochloride $(100 \mathrm{mg} / \mathrm{kg})$ and xylazine $(5 \mathrm{mg} / \mathrm{kg}$ ) given by intramuscular injection. Animals were subject to tracheotomy and artificially ventilated with isoflurane (2\% during surgery, $0.8 \%$ during recordings) in $\mathrm{O}_{2} / \mathrm{N}_{2} \mathrm{O}$ (30:70 ratio). Muscular relaxation was obtained by continuous injection of pancuronium bromide $(0.2 \mathrm{mg} / \mathrm{h})$ infused with $5 \%$ dextrose in a lactated Ringer's solution through an intraperitoneal cannula. End-tidal $\mathrm{CO}_{2}$, core body temperature, and electrocardiogram were continuously monitored to evaluate depth of anesthesia and the animal's health. Animals were positioned in a stereotaxic apparatus and bilateral craniotomies and durectomies were performed over the visual cortex. An imaging chamber was attached to the skull with dental cement. The cortical surface was stained with the VSD RH-1691 (Optical Imaging, $0.5 \mathrm{mg} / \mathrm{ml}$ in saline) for $90 \mathrm{~min}$ (Shoham et al., 1999). The cortex was then rinsed with saline and the chamber filled with silicone oil (Polydimethylsiloxane, 200 fluid, viscosity $350 \mathrm{cSt}$, Sigma-Aldrich) or agarose in saline (1\%). One or two lateral craniotomies were also performed to access the thalamus. Stimulation electrodes were lowered with an angle of $80^{\circ}$. At the end of each experiment, electrolytic lesions were made at each microstimulation electrode site. Animals were killed by sodium pentobarbital injection (Euthanyl, $1000 \mathrm{mg} /$ $\mathrm{kg}$ ) and transcardially perfused with phosphate buffered $0.9 \%$ saline, followed by phosphate-buffered $4 \%$ paraformaldehyde. Brains were removed, postfixed, cryoprotected in $30 \%$ sucrose in phosphate buffer, and then frozen. Coronal sections $(40 \mu \mathrm{m})$ were cut with a Leica cryostat, collected in PBS and stained with cresyl violet. Verification of the electrode track positions were made to confirm the location of stimulating electrodes.

Imaging. Images were recorded using a 12 bit CCD camera (1M60, Dalsa, configuration: $512 \times 512$ pixels resolution, frame rate of $110 \mathrm{~Hz}$ ) driven by the Imager 3001 system (Optical Imaging). The cortex was illuminated with a $630 \mathrm{~nm}$ light source and fluorescence signals were filtered with a $665 \mathrm{~nm}$ high-pass filter. Stimulation and blank trials were triggered by the first heartbeat spike generated after an interstimulus period of $30 \mathrm{~s}$. Stimulation trials were subtracted from blank trials to remove bleaching drift and potential fluorescence artifacts caused by heartbeats.

Visual and thalamic stimulation. For retinotopic mapping experiments, visual stimuli were generated with custom made software using the OpenGL library and back-projected by an LCD projector onto a $110 \times 80 \mathrm{~cm}$ translucent screen placed $28 \mathrm{~cm}$ in front of the animal. Stimuli consisted of bright vertical bars presented monocularly on a dark
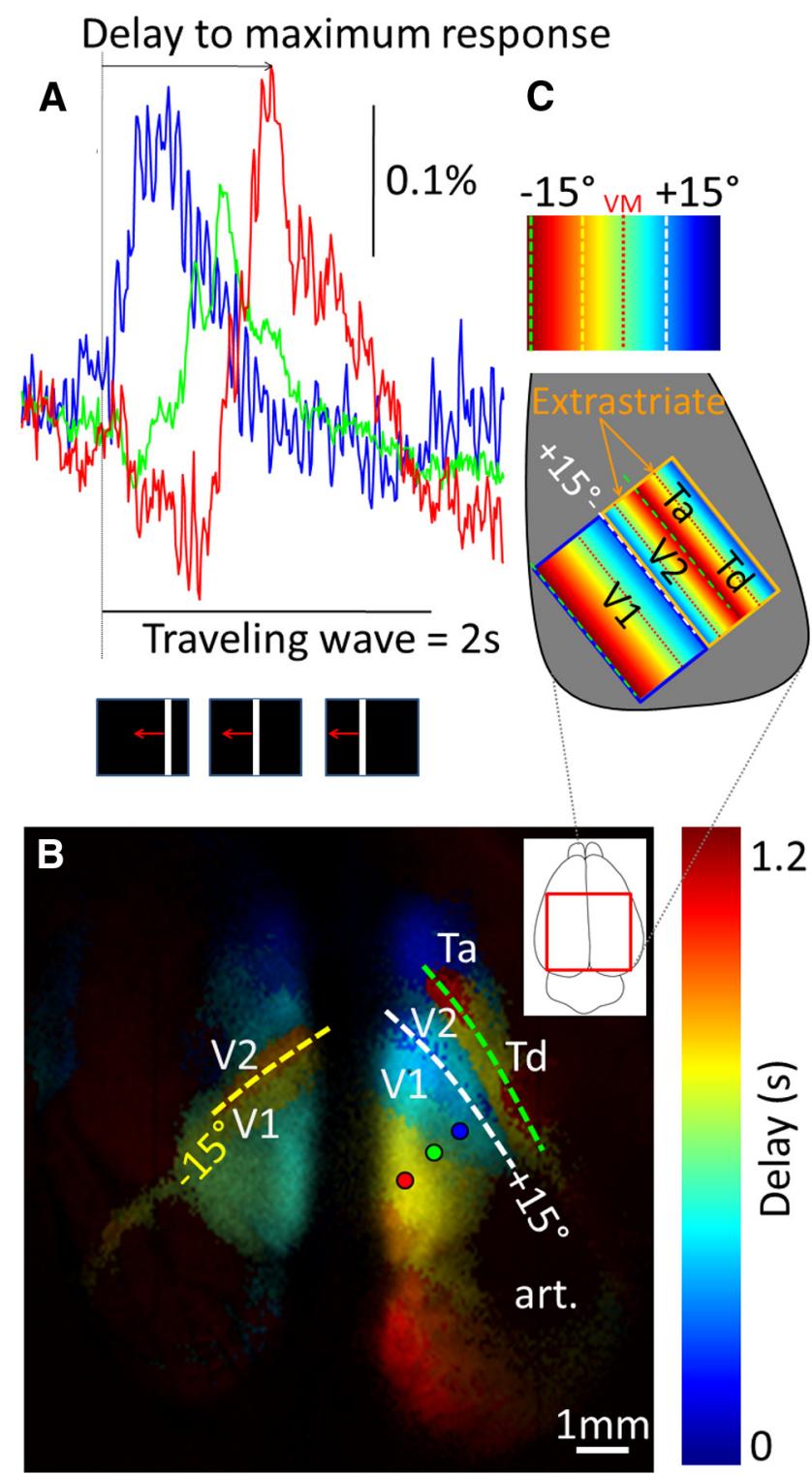

Figure 1. Retinotopic mapping. $\boldsymbol{A}$, Left, Responses to a moving bar recorded in the three cortical locations indicated in $\boldsymbol{B}$ (blue, green, and red circles). The bottom inset shows the visual stimulus. $\boldsymbol{B}$, Map of delay (time-to-peak) to reach the maximum response. Scale bar, $1 \mathrm{~mm}$. $\boldsymbol{C}$, Diagram showing the correspondence between visual field and the cortical retinotopy. Art, Artifact where the dye did not stain this location.

background (width: $2^{\circ}$, luminance: $80 \mathrm{~cd} / \mathrm{m}^{2}$ ). Stimuli were presented after a $500 \mathrm{~ms}$ baseline period. Data were averaged over 20 trials.

Electric microstimulations were performed in the dLGN and pulvinar nuclei. The pulvinar in tree shrew is comprised of three subnuclei, two of which [the ventral $(\mathrm{Pv})$ and caudal $(\mathrm{Pc})$ subnuclei] project to $\mathrm{V} 1$ and extrastriate cortex (Lyon et al., 2003a). These subnuclei were targeted in the present study. The accuracy of the stereotaxic dLGN location was confirmed by recording multiunit activity in response to contralateral and ipsilateral visual stimulation, because each of the six layers of the dLGN show preference for stimulus increments or decrements, ocular input, and retinotopic position (Conway and Schiller, 1983). Thus, electrodes were positioned in the dLGN to stimulate groups of neurons who's receptive-field locations matched the portion of $\mathrm{V} 1$ recorded by optical imaging (Bosking et al., 2000, 2002). Although, neurons in the dLGN have small, well defined receptive fields, pulvinar neuron receptive fields are diffuse and difficult to hand-map. Furthermore, crisp visual responses are unusual in the pulvinar; therefore, accurately locating this structure through electrophysiological recordings is more challenging. 


\section{A dLGN stimulation}
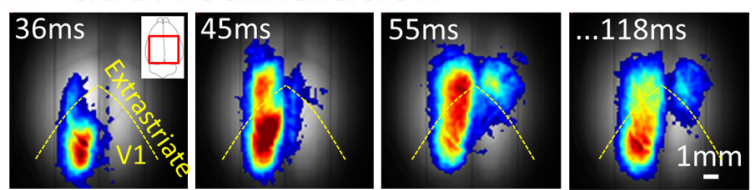

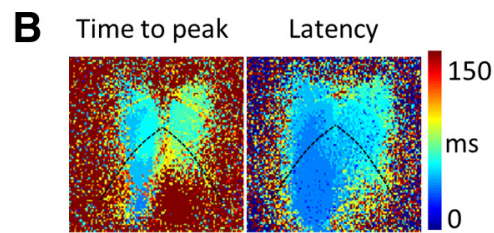

D

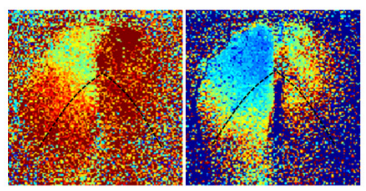

\section{E dLGN stimulation}

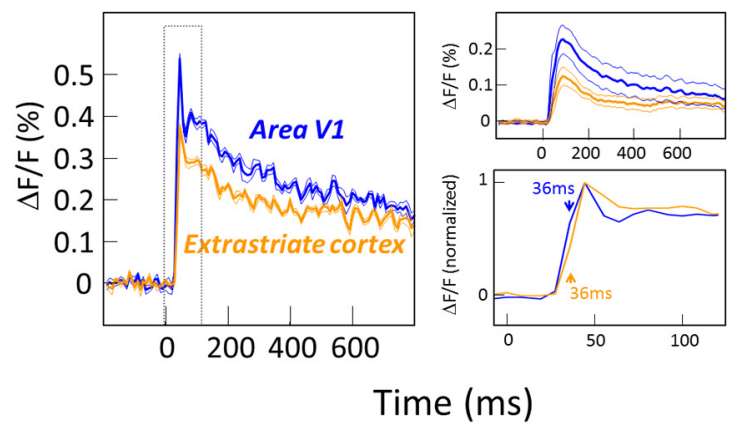

F Pulvinar stimulation

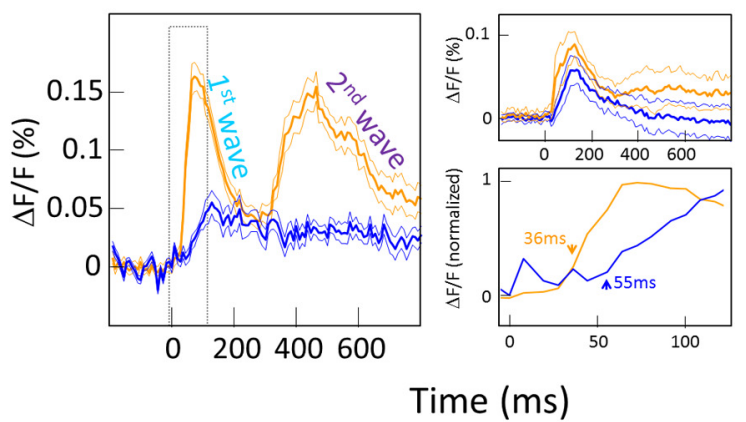

Figure 2. Cortical activation evoked by dLGN and pulvinar stimulation. $\boldsymbol{A}$, Example of averaged (between trials) cortical response maps (in color) overlaid on the basal fluorescence (in gray level) after stimulation of the left dLGN. $\boldsymbol{B}$, Time-to-peak and latency maps of responses evoked after dLGN stimulation. $\boldsymbol{C}, \boldsymbol{D}$, Same as $\boldsymbol{A}$ and $\boldsymbol{B}$ but for stimulation of the Pv subdivision of pulvinar. $\boldsymbol{E}$, Left, Fluorescence time course within area V1 (blue lines) and extrastriate cortex (thick orange lines) in response to dLGN stimulation. Right, Top, Averaged responses across recordings of the whole dataset. Right, Bottom, Normalized time close-up of the responses. Arrows indicate onset latency. $\boldsymbol{F}$, Same as $\boldsymbol{E}$ but for pulvinar stimulation.

Therefore, the location of the pulvinar electrode was determined by triangulation using the visuotopic position of dLGN multiunit receptive fields and their corresponding stereotaxic coordinates. In 28 sites of thalamic stimulation tested (from 5 animals), 15 were appropriately placed and generated cortical activation for $7 / 7 \mathrm{dLGN}$ and $7 / 8$ pulvinar stimulations (data were included only when responses exceeded by a factor of 2 the level of the SD of VSD signals). Cathodic electric microstimulations were produced with unipolar or bipolar tungsten electrodes (Microprobes). Stimulation trains followed Logothetis et al. (2010) and consisted in $100 \mathrm{~ms}$ train of $400.1 \mathrm{~ms}$ pulses of $250 \mu \mathrm{A}$ starting $200 \mathrm{~ms}$ after trial onset. Stimulations of the dLGN and pulvinar nuclei were pseudorandomly interleaved and repeated 5-53 times depending on the quality of the recordings. Retinotopic maps from four experiments were averaged and landmark-registered with the cranial bone sutures to create the logical masks delimiting V1 and extrastriate regions. To avoid possible signal contamination produced by inconsistencies in registration and/or in retinotopic map's resolution, masks were further eroded by $1 \mathrm{~mm}$ at the transition between V1 and extrastriate areas. Final masks were applied on the activated areas (pixel values above the mean $+1 \mathrm{SD}$ ), resulting in regions-of-interest (ROIs) used for quantifications. The onset latencies were calculated using a cumulative sum technique inspired from (Ouellette and Casanova, 2006). The SD of the signal variability during prestimulus period was calculated and the time at which the cumulative sum of the signal went $>3 \times \mathrm{SD}$ was considered to be the onset latency.

\section{Results}

Retinotopic organization of the tree shrew visual cortex

In the present study, cortical activity was recorded by measuring VSD fluorescence signals after thalamic or visual stimulation. To explore the impact of thalamic stimulation on the different cor- tical visual areas, retinotopic mapping experiments (Yang et al., 2009) were first performed to delimit the boundaries of primary visual and extrastriate cortical areas. In primates, the retinotopic organization of neurons is mirrored between each adjacent visual cortical area (Engel et al., 1994, 1997; DeYoe et al., 1996). In tree shrews, the retinotopic organization of area V1 has been characterized using intrinsic optical imaging techniques, but no maps could be obtained for extrastriate areas (Bosking et al., 2000, 2002). Unlike primates where the border between V1 and V2 occurs at the vertical meridian of the visual field, the transition between the two areas in tree shrew visual cortex takes place at $15^{\circ}$ in the ipsilateral visual field. In the present study, this transition zone was used to define the boundary between V1 and extrastriate regions. The retinotopic organization of the visual areas along the azimuth axis was assessed by measuring VSD response delays for each of the pixels in the image evoked by vertical white bars drifting across the visual field (Fig. 1). Assigning a lookup table to time-to-peak values yielded a delay map, revealing the retinotopic organization of cortical visual areas. In accordance with previous findings (Bosking et al., 2000), the transition from the $+15^{\circ}$ vertical meridian (Fig. 1, cyan) to the contralateral temporal visual field (red) was clearly observed along a posteromedial axis in V1. Neuronal activations were also observed outside area V1. An anterolateral adjacent area (likely area V2; Kaas, 2008) contained a mirrored representation of the V1 retinotopic pattern. Finally, additional cortical areas beyond V2, potentially corresponding to anterior temporal $(\mathrm{Ta})$ and dorsal temporal $(\mathrm{Td})$ areas (Wong and Kaas, 2009) that receive projections from the 
Boundaries

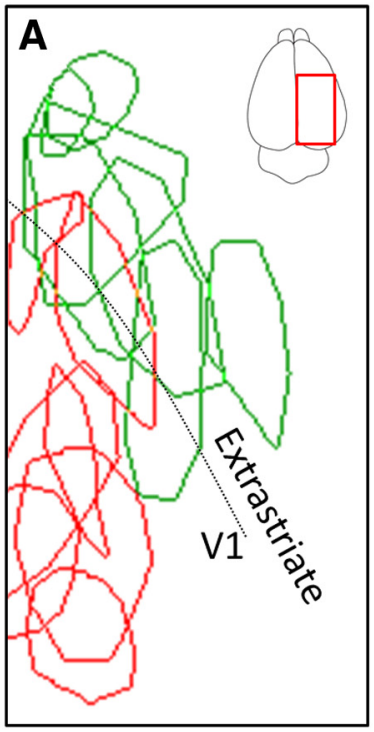

B
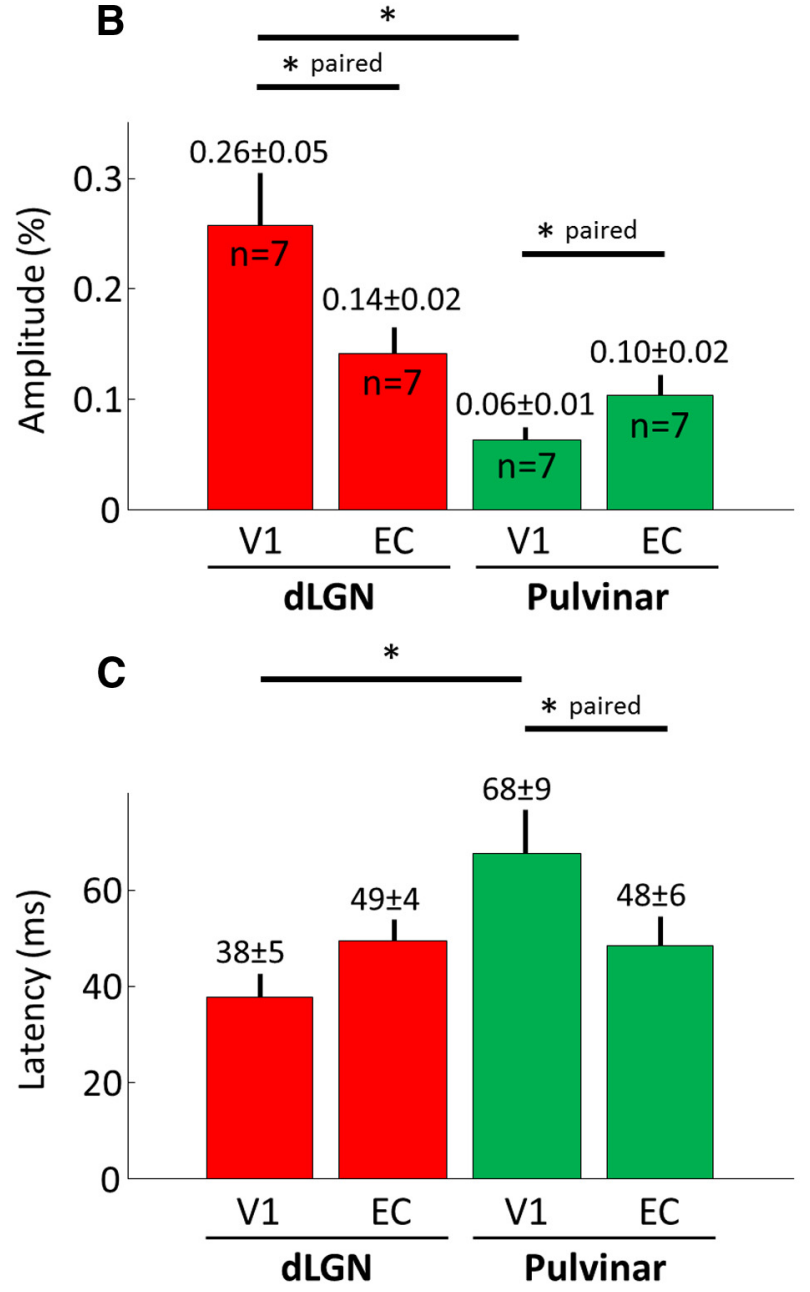

Figure 3. Properties of the cortical responses evoked by thalamic stimulation. $\boldsymbol{A}$, Left, Delimitations of the cortical surfaces activated in response to dLGN (red) and pulvinar nuclei (green) stimulation from all animals tested. Data originating from both hemispheres were pooled. Drawings delimit stimulated cortical areas based on the image immediately following response onset. Right, Crosses indicate centers of each ROI presented on the left. B, C, Amplitude and latencies measured in area V1 and extrastriate cortical areas after pulvinar and dLGN stimulations ( \pm SEM across values and number of observation). ${ }^{*} p<0.05, E C$, Extrastriate cortex. dorsal pulvinar (Pd) and Pc (Chomsung et al., 2010) were also retinotopically activated. This is the first time, using optical imaging techniques, that the retinotopic organization of cortical visual areas beyond area V1 has been functionally demonstrated in tree shrews. Thus, the use of VSD is a promising experimental protocol for the exploration of functional properties of extrastriate cortical areas in tree shrews.

\section{Cortical responses to thalamic stimulation}

Once V1 and extrastriate areas were defined based on retinotopic mapping, we explored the impact of dLGN or pulvinar stimulation on activity patterns in these areas. Examples and group data of VSD cortical responses to thalamic stimulation are shown in Figures 2 and 3, respectively. Stimulation of the dLGN produced responses that were initially located in area V1 (Figs. $2 A, 3 A$, red), and then spread to extrastriate areas. In addition, dLGN stimulation also activated cortical regions of the opposite hemisphere, supporting the hypothesis that electric microstimulation can efficiently induce polysynaptic transmission (Logothetis et al., 2010). The amplitudes of V1 responses to dLGN stimulation were larger than extrastriate responses (Wilcoxon signed rank test, $p=$ 0.031 ; Fig. $3 B$ ), and activation of extrastriate areas by dLGN stimulation was always preceded by activation of area V1 (although no significant difference was detected when the latencies of V1 and extrastriate responses were compared, $p=0.063$; Fig. $3 C$ ).

In contrast, the VSD response amplitudes in extrastriate areas evoked by pulvinar stimulation (Figs. $2 C, 3 A$, green) were larger and faster than those evoked in V1 (Fig. $3 B, C ; p=0.047$ and $p=$ 0.031 for amplitude and latency, respectively). Notably, stimulation of the Pc evoked more lateral cortical responses (likely in area $\mathrm{Td}$ ) compared with stimulation of the $\mathrm{Pv}$, which evoked more anterior responses, (likely in area Ta, data not shown), reflecting anatomical connections patterns.

No difference was observed in response latency (Wilcoxon rank sum test, $p=0.830)$ or amplitude $(p=0.259)$ in extrastriate areas following dLGN or pulvinar stimulation. However in V1, shorter latencies $(p=0.019)$ and higher response amplitudes $(p=0.001)$ were obtained with dLGN stimulation when compared with pulvinar stimulation. Thus, although stimulation of the pulvinar was able to drive responses in extrastriate areas with a strength similar to that following stimulation of the dLGN, its effect on V1 activity was more subtle. Interestingly, the activation observed in extrastriate areas was often biphasic [second/first wave amplitude ratio: $0.17 \pm 0.08(n=13)$ and $0.65 \pm 0.14(n=$ $12)$ in V1 and extrastriate areas, respectively, $p=0.004$ ], with a second wave occurring $>666 \pm 49 \mathrm{~ms}$ after the initial wave. Although not significant, this phenomenon was found to occur slightly more often after stimulation of pulvinar than after stimulation of the dLGN.

\section{Discussion}

In the present study, an accurate spatiotemporal comparison between cortical responses evoked by activation of different thalamic nuclei was made possible by the combination of different experimental strategies: (1) VSD imaging was used to monitor cortical activity (Shoham et al., 1999). This technique provides a high spatiotemporal resolution measure of subthreshold and suprathreshold activity of cortical neurons, mostly pyramidal cells (Chemla and Chavane, 2010). (2) Localized stimulation was made possible by the use of electrical microstimulation ( $\mathrm{Su}$ zurikawa et al., 2009; Logothetis et al., 2010; Brock et al., 2013). (3) Finally, this innovative experimental approach was made possible by the use of tree shrews, considered to be closet relatives of 
primates, which possess a fully flat cortex devoid of any sulci. Because of this, the continuous surface of cortex could be explored with much more precision than alternative approaches that lack high spatiotemporal resolution, such as fMRI BOLD signals (Logothetis et al., 2010).

Another major advance presented in this study was the ability to image the activity in extrastriate areas in tree shrews using VSD imaging. The retinotopic mapping approach used permitted the precise delimitation of several extrastriate cortical areas. In primates and cats, the visual field representation in each adjacent cortical area is mirrored and the borders between areas generally correspond to azimuth limits of the visual field (Engel et al., 1994, 1997; DeYoe et al., 1996). Conventional methods to identify retinotopic maps are not suited to VSD imaging, because of the possible phototoxicity associated with prolonged illumination. The use of response delay maps provided a valuable alternative to obtain high resolution retinotopic maps while reducing the time of illumination (Yang et al., 2009). Although the use of moving bars is known to induce anticipatory responses generated at early retinal stages (Berry et al., 1999), this putative offset delay in the response appeared to be constant, considering that it did not prevent observation of gradient and phase maps in intrinsic imaging studies (Kalatsky and Stryker, 2003; Vanni et al., 2010), including the present study.

The main finding of this study was that the activation of firstorder and high-order thalamic nuclei yields distinct spatiotemporal patterns of activity in visual cortical areas. Stimulation of the dLGN produced "driver-type" responses in area V1 but also consistently activated extrastriate cortices with similar latencies. In mammals, dLGN-induced extrastriate activation could result from three potential synaptic circuits: corticocortical (Sesma et al., 1984; Lyon et al., 1998) and cortico-thalamo-cortical feedforward connections (Bender, 1983; Casanova et al., 1997; Hilgetag et al., 2000a,b; Rushmore et al., 2005; Huppé-Gourgues et al., 2006) or from direct geniculo-cortical connections (Kawano, 1998; Lyon et al., 2003b; Sincich et al., 2004). Unfortunately, given the temporal resolution ( $9 \mathrm{~ms} /$ frame) of our data acquisition, the functional contribution of corticocortical and corticothalamo-cortical synaptic pathways following dLGN stimulation cannot be determined. In contrast to the dLGN, pulvinar stimulation induced responses mainly in extrastriate cortex. This result is likely due to the presence of dense projections from the pulvinar nucleus to layer IV of the extrastriate cortex (Chomsung et al., 2010). The weak responses in area V1 induced by pulvinar stimulation could result from two anatomical pathways: a direct pulvinar projection to striate cortex (Raczkowski and Rosenquist, 1983; Abramson and Chalupa, 1985; Lyon et al., 2003b) or corticocortical feedback connections from extrastriate cortex to V1 (Sesma et al., 1984; Lyon et al., 1998). The slow kinetics of V1 activation following pulvinar stimulation, suggests that the pulvinar nucleus primarily affects V1 activity via activation of feedback projections from extrastriate areas to V1. This hypothesis is in accordance with recent findings showing that pulvinar inactivation changed gain response modulation and orientation tuning of V1 neurons in galagos (Purushothaman et al., 2012). Future experimental protocols using concurrent visual and pulvinar stimulation could help to delineate the functional contribution of the pulvinar nucleus in shaping spatiotemporal activity patterns of V1 neurons.

Overall, the present data provide evidence for two concurrent thalamocortical bottom-up pathways targeting higher-level cortical areas. Our results show that a geniculo-cortical pathway drives a fast flow of information from area V1 to extrastriate areas, whereas the pulvinocortical pathway bypasses V1 and directly drives extrastriate areas. Although the pulvinar does not receive a strong retinal input, it receives prominent projections from V1, extrastriate cortex, and the superior colliculus (Chomsung et al., 2008, 2010). The pulvinar pathway may thus both permit cortico-thalamo-cortical communication of visual signals (Casanova, 2004) and also inform the visual cortex about superior colliculus activity controlling eye and head movements (Sherman and Guillery, 2011).

Interestingly, we observed a surprising difference in the later part of the cortical responses evoked by thalamic stimulation; within extrastriate areas, but not within V1, a larger second delayed depolarization wave was observed following stimulation of either the dLGN or the pulvinar nucleus. Dissimilarities in recurrent excitatory and inhibitory circuits within V1 and extrastriate areas could explain these timing differences as observed in the somatosensory cortex (Derdikman et al., 2003; Petersen et al., 2003). Future investigations comparing direct cortical stimulation with thalamic stimulation may help to unravel the origins of the second wave of activity that we observed in extrastriate areas.

In conclusion, this study presented a novel way to explore thalamocortical interactions by combining functional highresolution imaging and microstimulation. We observed VSD responses in tree shrews not previously described, and demonstrated that in the striate cortex the effects of stimulating the dLGN or pulvinar nucleus were distinct, with respective characteristics consistent with driving and modulatory effects on $\mathrm{V} 1$ activity patterns.

\section{References}

Abramson B, Chalupa LM (1985) The laminar distribution of cortical connections with the tecto- and cortico-recipient zones in the cat's lateral posterior nucleus. Neuroscience 15:81-95. CrossRef Medline

Bender DB (1983) Visual activation of neurons in the primate pulvinar depends on cortex but not colliculus. Brain Res 279:258-261. CrossRef Medline

Berry MJ 2nd, Brivanlou IH, Jordan TA, Meister M (1999) Anticipation of moving stimuli by the retina. Nature 398:334-338. CrossRef Medline

Bosking WH, Kretz R, Pucak ML, Fitzpatrick D (2000) Functional specificity of callosal connections in tree shrew striate cortex. J Neurosci 20:23462359. Medline

Bosking WH, Crowley JC, Fitzpatrick D (2002) Spatial coding of position and orientation in primary visual cortex. Nat Neurosci 5:874-882. CrossRef Medline

Brock AA, Friedman RM, Fan RH, Roe AW (2013) Optical imaging of cortical networks via intracortical microstimulation. J Neurophysiol 110: 2670-2678. CrossRef Medline

Casanova C (2004) The visual function of the pulvinar. In: The visual neurosciences (Werner JS, Chalupa LM, eds). Cambridge, MA: MIT.

Casanova C, Savard T, Darveau S (1997) Contribution of area 17 to cell responses in the striate-recipient zone of the cat's lateral posteriorpulvinar complex. Eur J Neurosci 9:1026-1036. CrossRef Medline

Chemla S, Chavane F (2010) A biophysical cortical column model to study the multi-component origin of the VSDI signal. Neuroimage 53:420438. CrossRef Medline

Chomsung RD, Petry HM, Bickford ME (2008) Ultrastructural examination of diffuse and specific tectopulvinar projections in the tree shrew. J Comp Neurol 510:24-46. CrossRef Medline

Chomsung RD, Wei H, Day-Brown JD, Petry HM, Bickford ME (2010) Synaptic organization of connections between the temporal cortex and pulvinar nucleus of the tree shrew. Cereb Cortex 20:997-1011. CrossRef Medline

Conway JL, Schiller PH (1983) Laminar organization of tree shrew dorsal lateral geniculate nucleus. J Neurophysiol 50:1330-1342. Medline

Derdikman D, Hildesheim R, Ahissar E, Arieli A, Grinvald A (2003) Imaging spatiotemporal dynamics of surround inhibition in the barrels somatosensory cortex. J Neurosci 23:3100-3105. Medline

DeYoe EA, Carman GJ, Bandettini P, Glickman S, Wieser J, Cox R, Miller D, 
Neitz J (1996) Mapping striate and extrastriate visual areas in human cerebral cortex. Proc Natl Acad Sci U S A 93:2382-2386. CrossRef Medline

Engel SA, Rumelhart DE, Wandell BA, Lee AT, Glover GH, Chichilnisky EJ, Shadlen MN (1994) fMRI of human visual cortex. Nature 369:525. CrossRef Medline

Engel SA, Glover GH, Wandell BA (1997) Retinotopic organization in human visual cortex and the spatial precision of functional MRI. Cereb Cortex 7:181-192. CrossRef Medline

Hilgetag CC, O'Neill MA, Young MP (2000a) Hierarchical organization of macaque and cat cortical sensory systems explored with a novel network processor. Philos Trans R Soc Lond B Biol Sci 355:71-89. CrossRef Medline

Hilgetag CC, Burns GA, O’Neill MA, Scannell JW, Young MP (2000b) Anatomical connectivity defines the organization of clusters of cortical areas in the macaque monkey and the cat. Philos Trans R Soc Lond B Biol Sci 355:91-110. CrossRef Medline

Huppé-Gourgues F, Bickford ME, Boire D, Ptito M, Casanova C (2006) Distribution, morphology, and synaptic targets of corticothalamic terminals in the cat lateral posterior-pulvinar complex that originate from the posteromedial lateral suprasylvian cortex. J Comp Neurol 497:847-863. CrossRef Medline

Kaas JH (2008) The evolution of the complex sensory and motor systems of the human brain. Brain Res Bull 75:384-390. CrossRef Medline

Kalatsky VA, Stryker MP (2003) New paradigm for optical imaging: temporally encoded maps of intrinsic signal. Neuron 38:529-545. CrossRef Medline

Kawano J (1998) Cortical projections of the parvocellular laminae C of the dorsal lateral geniculate nucleus in the cat: an anterograde wheat germ agglutinin conjugated to horseradish peroxidase study. J Comp Neurol 392:439-457. CrossRef Medline

Logothetis NK, Augath M, Murayama Y, Rauch A, Sultan F, Goense J, Oeltermann A, Merkle H (2010) The effects of electrical microstimulation on cortical signal propagation. Nat Neurosci 13:1283-1291. CrossRef Medline

Lyon DC, Jain N, Kaas JH (1998) Cortical connections of striate and extrastriate visual areas in tree shrews. J Comp Neurol 401:109-128. CrossRef Medline

Lyon DC, Jain N, Kaas JH (2003a) The visual pulvinar in tree shrews: I. Multiple subdivisions revealed through acetylcholinesterase and Cat-301 chemoarchitecture. J Comp Neurol 467:593-606. CrossRef Medline

Lyon DC, Jain N, Kaas JH (2003b) The visual pulvinar in tree shrews: II. Projections of four nuclei to areas of visual cortex. J Comp Neurol 467: 607-627. CrossRef Medline

Malpeli JG, Schiller PH, Colby CL (1981) Response properties of single cells in monkey striate cortex during reversible inactivation of individual lateral geniculate laminae. J Neurophysiol 46:1102-1119. Medline

Ogren MP, Hendrickson AE (1977) The distribution of pulvinar terminals in visual areas 17 and 18 of the monkey. Brain Res 137:343-350. CrossRef Medline
Ouellette BG, Casanova C (2006) Overlapping visual response latency distributions in visual cortices and LP-pulvinar complex of the cat. Exp Brain Res 175:332-341. CrossRef Medline

Petersen CC, Grinvald A, Sakmann B (2003) Spatiotemporal dynamics of sensory responses in layer $2 / 3$ of rat barrel cortex measured in vivo by voltage-sensitive dye imaging combined with whole-cell voltage recordings and neuron reconstructions. J Neurosci 23:1298-1309. Medline

Purushothaman G, Marion R, Li K, Casagrande VA (2012) Gating and control of primary visual cortex by pulvinar. Nat Neurosci 15:905-912. CrossRef Medline

Raczkowski D, Rosenquist AC (1983) Connections of the multiple visual cortical areas with the lateral posteror-pulvinar complex and adjacent thalamic nuclei in the cat. J Neurosci 3:1912-1942. Medline

Rockland KS, Andresen J, Cowie RJ, Robinson DL (1999) Single axon analysis of pulvinocortical connections to several visual areas in the macaque. J Comp Neurol 406:221-250. CrossRef Medline

Rushmore RJ, Payne BR, Lomber SG (2005) Functional impact of primary visual cortex deactivation on subcortical target structures in the thalamus and midbrain. J Comp Neurol 488:414-426. CrossRef Medline

Sesma MA, Casagrande VA, Kaas JH (1984) Cortical connections of area 17 in tree shrews. J Comp Neurol 230:337-351. CrossRef Medline

Sherman SM, Guillery RW (2011) Distinct functions for direct and transthalamic corticocortical connections. J Neurophysiol 106:1068-1077. CrossRef Medline

Shoham D, Glaser DE, Arieli A, Kenet T, Wijnbergen C, Toledo Y, Hildesheim R, Grinvald A (1999) Imaging cortical dynamics at high spatial and temporal resolution with novel blue voltage-sensitive dyes. Neuron 24:791-802. CrossRef Medline

Sincich LC, Park KF, Wohlgemuth MJ, Horton JC (2004) Bypassing V1: a direct geniculate input to area MT. Nat Neurosci 7:1123-1128. CrossRef Medline

Suzurikawa J, Tani T, Nakao M, Tanaka S, Takahashi H (2009) Voltagesensitive-dye imaging of microstimulation-evoked neural activity through intracortical horizontal and callosal connections in cat visual cortex. J Neural Eng 6:066002. CrossRef Medline

Vanni MP, Provost J, Lesage F, Casanova C (2010) Evaluation of receptive field size from higher harmonics in visuotopic mapping using continuous stimulation optical imaging. J Neurosci Methods 189:138-150. CrossRef Medline

Warner CE, Goldshmit Y, Bourne JA (2010) Retinal afferents synapse with relay cells targeting the middle temporal area in the pulvinar and lateral geniculate nuclei. Front Neuroanat 4:8. CrossRef Medline

Wong P, Kaas JH (2009) Architectonic subdivisions of neocortex in the tree shrew (Tupaia belangeri). Anat Rec (Hoboken) 292:994-1027. CrossRef

Yang Z, Heeger DJ, Seidemann E (2007) Rapid and precise retinotopic mapping of the visual cortex obtained by voltage-sensitive dye imaging in the behaving monkey. J Neurophysiol 98:1002-1014. CrossRef Medline 